\title{
SEM, TEM, and Cryo-EM of Electrodeposited Li, Mg, and Ca Metal Electrodes
}

Katherine Jungjohann ${ }^{1 *}$, Katharine Harrison ${ }^{2}$, Subrahmanyam Goriparti ${ }^{2}$, Paul Cullier ${ }^{2}$, Robert Fischer ${ }^{3}$, Brad Boyce ${ }^{1}$, Zachary Casias ${ }^{4}$, Nathan Hahn ${ }^{2}$, Brian Perdue ${ }^{5}$, and Kevin Zavadil ${ }^{2}$

1. Center for Integrated Nanotechnologies, Sandia National Laboratories, Albuquerque, USA.

2. Nanoscale Sciences Department, Sandia National Laboratories, Albuquerque, USA.

3. CAMCOR Facility, University of Oregon, Eugene, USA.

4. Materials Mechanics \& Tribology, Sandia National Laboratories, Albuquerque, USA.

5. Power Sources Research and Development, Sandia National Laboratories, Albuquerque, USA.

* Corresponding author: kljungj@sandia.gov

Over the past seven years, significant improvements with in-situ electrochemical scanning/transmission electron microscopy (S/TEM) platforms have allowed for the real-time observation of nanoscale reactions on battery electrodes [1]. The field has advanced from uncontrolled electrochemical potentials, into quantitative charge cycling [2], analytical characterization during charge cycling [3], and correlated structure-electrochemical relationship determination [4,5]. These advancements have been useful in gaining a deeper understanding of the electrochemical plating and stripping of metals ( $\mathrm{Li}, \mathrm{Mg}$ and $\mathrm{Ca}$ ) that are among the highest theoretical energy storage capacity electrodes available for future portable batteries. The in-situ STEM insights thus far gained on Li electrodeposition and dissolution have indicated that electrochemical S/TEM platforms fall short in the ability to replicate the electrode contact pressure that is applied in general battery testing platforms (coin cells) [6]. Electrochemical S/TEM platforms are configured with electrodes patterned in a planar geometry on the SiN membrane windows, as imaging is performed on individual electrodes instead of stacked electrodes. Therefore, this limitation produces electrodeposited morphologies that are not representative of the structures observed in coin cells.

In order to use electron microscopy to precisely characterize the structure and composition of electrode materials from realistic battery testing conditions, we have turned to using the ex-situ approach demonstrated by Zachman et al. [7]. Cryogenic temperature electron microscopy can provide the stability needed to image beam-sensitive Li structures, create focused ion-beam cross-sections for S/TEM analysis, and to collect analytical maps from the electron transparent lamella and cross-sectional samples. These methods have been explored to gain deeper insights into the electrodeposition of $\mathrm{Li}, \mathrm{Mg}$, and $\mathrm{Ca}$ structures, with an emphasis in retaining the surface structure on the electrodes (including electrolyte and separator contact). Sample preparation techniques will be discussed in this presentation, for obtaining SEM and S/TEM cross-sectional images from metal electrode surfaces.

Our early data on using these approaches suggests that there is a range of surface contact pressures that have a strong impact on the resulting electrodeposited Li morphology during cycling. Li morphology has been observed with cryo-SEM imaging (Figure 1) to change relative to the cycle number and contact pressure. The details of these changes will be presented. Additionally, details about the characteristics of the surface compositions for $\mathrm{Li}, \mathrm{Mg}$, and $\mathrm{Ca}$ metal electrodes will be discussed. This ex-situ characterization methodology provides the means to understand the critical factors in controlling electrodeposition of promising metal anode electrodes for future portable energy storage technologies [8]. 
References:

[1] MJ Williamson et al., Nat. Mater. 2 (2003), p. 532.

[2] RR Unocic et al., Microsc. Microanal. 20 (2014), p. 452.

[3] ME Holtz et al., Nano Letters 14 (2014), p. 1453.

[4] AJ Leenheer et al., ACS Nano 9 (2015), p. 4379.

[5] AJ Leenheer, KL Jungjohann, KR Zavadil, and CT Harris, ACS Nano 10 (2016), p. 5670.

[6] KL Harrison et al., ACS Nano 11 (2017), p. 11194.

[7] MJ Zachman et al., Nature 560 (2018), p. 345.

[8] A portion of this work was supported by the Joint Center for Energy Storage Research (JCESR), an Energy Innovation Hub funded by the U.S. Department of Energy, Office of Science, Office of Basic Energy Sciences. A portion of this work was supported by the Laboratory Directed Research and Development Program at Sandia National Laboratories. This work was performed, in part, at the Center for Integrated Nanotechnologies, an Office of Science User Facility operated for the U.S. Department of Energy (DOE) Office of Science. Sandia National Laboratories is a multi-mission laboratory managed and operated by National Technology and Engineering Solutions of Sandia, LLC., a wholly owned subsidiary of Honeywell International, Inc., for the U.S. DOE's National Nuclear Security Administration under contract DE-NA-0003525. The views expressed in the article do not necessarily represent the views of the U.S. DOE or the United States Government.
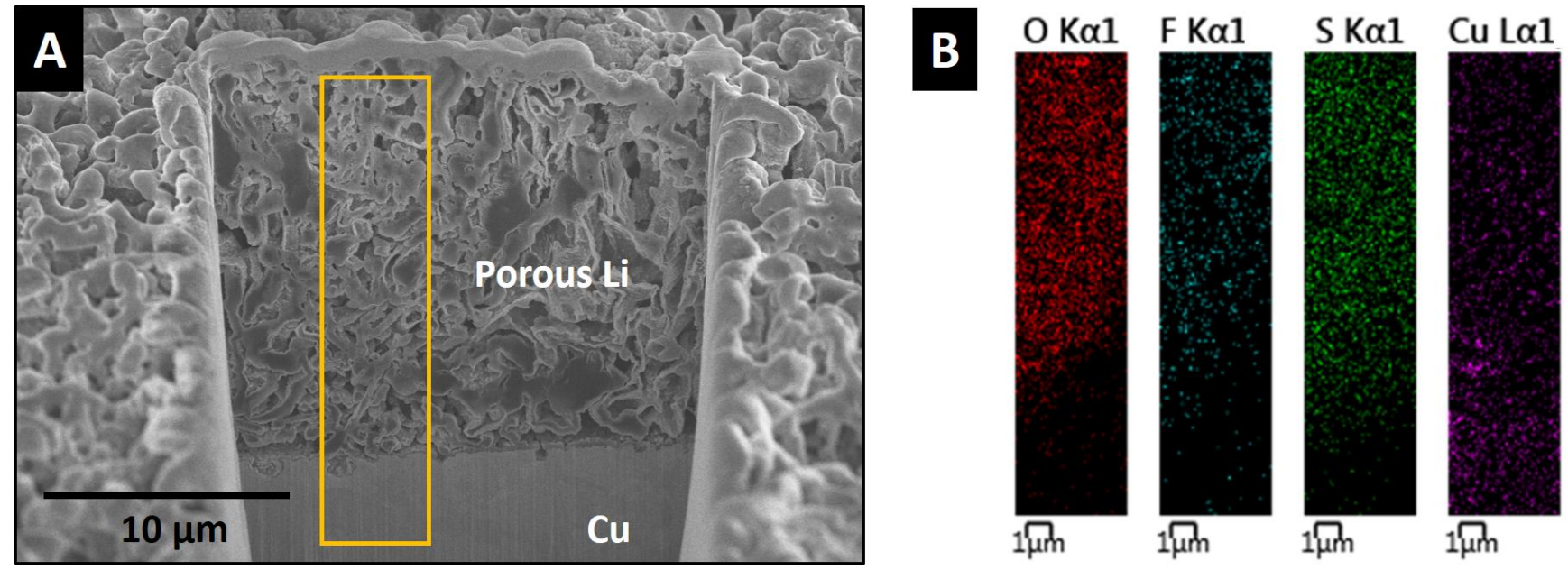

Figure 1. Scanning Electron Microscopy data from a focus ion beam milled cross-section of a dried Li metal electrode on a copper current collector at liquid nitrogen temperature. A) SEM image, the yellow region indicating the region of interest, B) electron $\mathrm{X}$-ray spectroscopy maps of elements $\mathrm{O}, \mathrm{F}, \mathrm{S}$, and $\mathrm{Cu}$ from the region of interest of $\mathrm{Li}$ metal plated vs. a $\mathrm{Li}$ metal counter electrode in $4 \mathrm{M}$ lithium bis(fluorosulfulfonyl)imide in dimethoxyethane. 